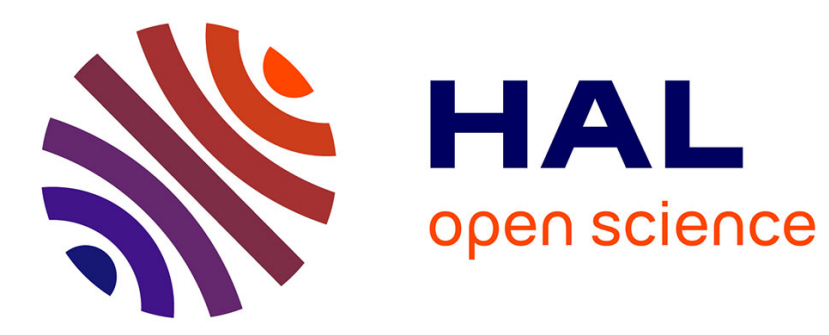

\title{
Wideband Dual-Polarized Full-Duplex Antenna Array
}

Hadi Hijazi, Marc Le Roy, Raafat Lababidi, Denis Le Jeune, André Pérennec

\section{To cite this version:}

Hadi Hijazi, Marc Le Roy, Raafat Lababidi, Denis Le Jeune, André Pérennec. Wideband DualPolarized Full-Duplex Antenna Array. International Conference on Advanced Technologies for Communications (ATC'21), Oct 2021, Ho Chi Minh city, Vietnam. pp.1-6. hal-03428804

\section{HAL Id: hal-03428804 \\ https://hal.univ-brest.fr/hal-03428804}

Submitted on 15 Nov 2021

HAL is a multi-disciplinary open access archive for the deposit and dissemination of scientific research documents, whether they are published or not. The documents may come from teaching and research institutions in France or abroad, or from public or private research centers.
L'archive ouverte pluridisciplinaire HAL, est destinée au dépôt et à la diffusion de documents scientifiques de niveau recherche, publiés ou non, émanant des établissements d'enseignement et de recherche français ou étrangers, des laboratoires publics ou privés. 


\title{
Wideband Dual-Polarized Full-Duplex Antenna Array
}

\author{
Hadi Hijazi ${ }^{1,2^{*}}$, Marc Le Roy ${ }^{2}$, Raafat Lababidi ${ }^{1}$, Denis Le Jeune ${ }^{1}$, Andre Pérennec ${ }^{2}$ \\ ${ }^{1}$ ENSTA Bretagne, Lab-STICC, CNRS, UMR 6285, F-29200 Brest, France \\ ${ }^{2}$ Univ Brest, Lab-STICC, CNRS, UMR 6285, F-29200 Brest, France \\ * hadi.hijazi@ensta-bretagne.org
}

(Invited Paper)

\begin{abstract}
In this paper, a wideband dual-polarized fullduplex antenna array is presented. The array is formed of four dual-polarized Vivaldi antennas with a modified feeding to obtain total symmetry between the orthogonal polarizations. The array is fed by a wideband 4-way balun that can split the input signal into four output signals of equal amplitudes with two of them being phase inverted with respect to the other two. The simulated results indicate that the array is matched from 2 $\mathrm{GHz}$ to beyond $20 \mathrm{GHz}$ with an average gain of $11 \mathrm{dBi}$. Also, under ideal conditions, the array can achieve a high level of selfinterference cancellation ( $\geq 120 \mathrm{~dB}$ ), and this level is estimated to be better than $40 \mathrm{~dB}$ in practice.
\end{abstract}

Keywords-dual-polarized, full-duplex, microstrip-to-slotline transition, wideband, Vivaldi antenna.

\section{INTRODUCTION}

For a long time, it was assumed that two wireless radios cannot communicate on the same frequency at the same time. This is due to the high-power signals coupled from the transmitter of the local radio to its own receiver, which results in a strong self-interference that jams the weaker signal received from the distant radio. Up till now, self-interference was avoided by separating the transmitted signals from the received signals by using orthogonal time and/or frequency resources. Such mode of communication is called out-of-band full-duplex and is limited by the number of frequencies available in a certain band, which are finite. To reduce this limitation, a huge effort was invested in achieving an in-band full-duplex communication, where both radios can transmit and receive on the same frequency at the same time, which can theoretically double the available frequency resources in a certain band. In-band full-duplex manages to achieve simultaneous transmission/reception by employing various cancellation techniques to reduce self-interference to below the receiver's noise floor, such that it does not affect the receiver's signal-to-noise ratio. And it was found by a study made in the $\mathrm{Wi}-\mathrm{Fi}$ band that a cancellation level of $110 \mathrm{~dB}$ can be sufficient to achieve an in-band full-duplex communication [1]. The cancellation circuitry can be implemented at multiple stages of the radio front-end starting from the antennas and passing through the analog domain to the digital domain.

The simultaneous transmit-receive property of in-band full-duplex technology not only doubles the spectral resources, but also it can enable the introduction of novel and efficient multiple access techniques, physical layer security protocols, relaying solutions, and can reduce air interface delay. Also, it can benefit radar systems, cognitive radios (CR), and multiple-input multiple output (MIMO) arrays. These potential applications and possibilities span different bands of the spectrum, so it is highly valuable to have some wideband full-duplex systems that can serve all these applications instead of designing separate systems dedicated for each individual application. However, while it is possible to achieve wideband cancellation at the antenna level, reaching such wideband in the analog and digital domains is still problematic due to circuitry bandwidth limitations and cost. So, the scope of this work will focus on wideband cancellation techniques at the antenna level.

Several techniques were proposed to achieve wideband cancellation at the antenna level. For monostatic systems, some implementations based on wideband circulators and hybrid transformers can be found in [2] and [3], respectively. On the other hand, for bistatic systems, cancellation can be achieved by increasing antenna separation distance [4], directing antenna beams in different directions [4], near-field cancellation [5], circularly phased arrays [6], and placing high impedance structures between the antennas [7]. Mainly, nearfield cancellation has shown an impressive capability of achieving high levels of cancellation with minimum system size and simple design, and without affecting the radiation performance of the system.

Having wideband dual-polarized full-duplex antenna systems can be attractive for several applications. For instance, dual-polarized antennas can be used in radar systems for better spatial recognition of the scanned targets, and they can also be used in mobile and vehicular applications to ensure that the user can still receive signals properly even during its motion. Some implementations of dual-polarized full-duplex antennas are available in the literature [8]-[10]. Almost all of referenced works use the near-field cancellation technique, which is conveniently compatible with dual-polarized configurations, however, all the presented works are narrowband. So, in the light of the above, in this work we propose a wideband dual-polarized full-duplex array which is also based on the near-field cancellation technique. The array consists of four all-metal Vivaldi antennas and two wideband baluns based on microstrip-to-slotline transitions. All the array components were modeled and simulated in CST Microwave Studio. Based on that, the remaining part of this paper will be organized as follows: the proposed array with its components will be presented in Section II, then its performance will be presented in Section III, and finally, Section IV will conclude and point to future perspectives.

\section{ARRAY DESIGN}

The wideband full-duplex array is based on the near-field cancellation technique, which is discussed in [5]. The array is formed of four dual-polarized Vivaldi antennas as shown in Figure 1(a), and each antenna is sequentially rotated by $90^{\circ}$ with respect to the others. Thus, we obtain two pairs of diametrically opposite antennas: one pair will be used to transmit while the other pair will be used to receive. If signals of equal amplitudes and opposite phases are injected in each pair of opposite antennas, as in Figure 1(b), then their radiated 


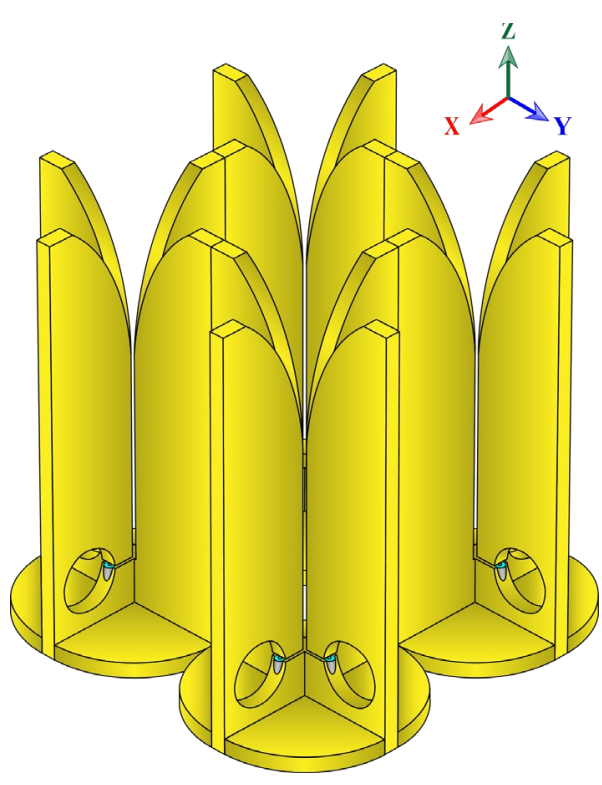

(a)

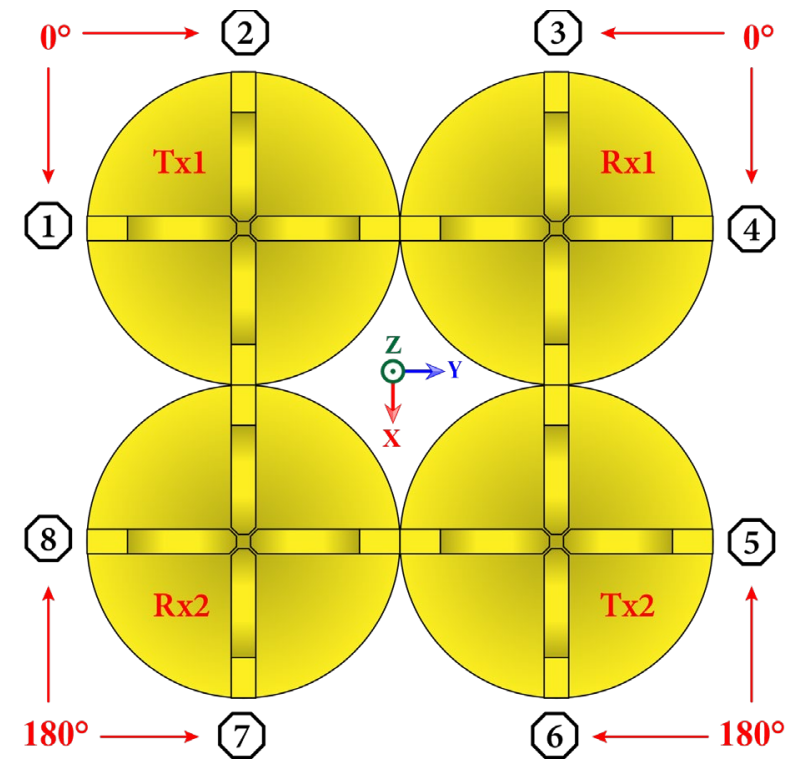

(b)

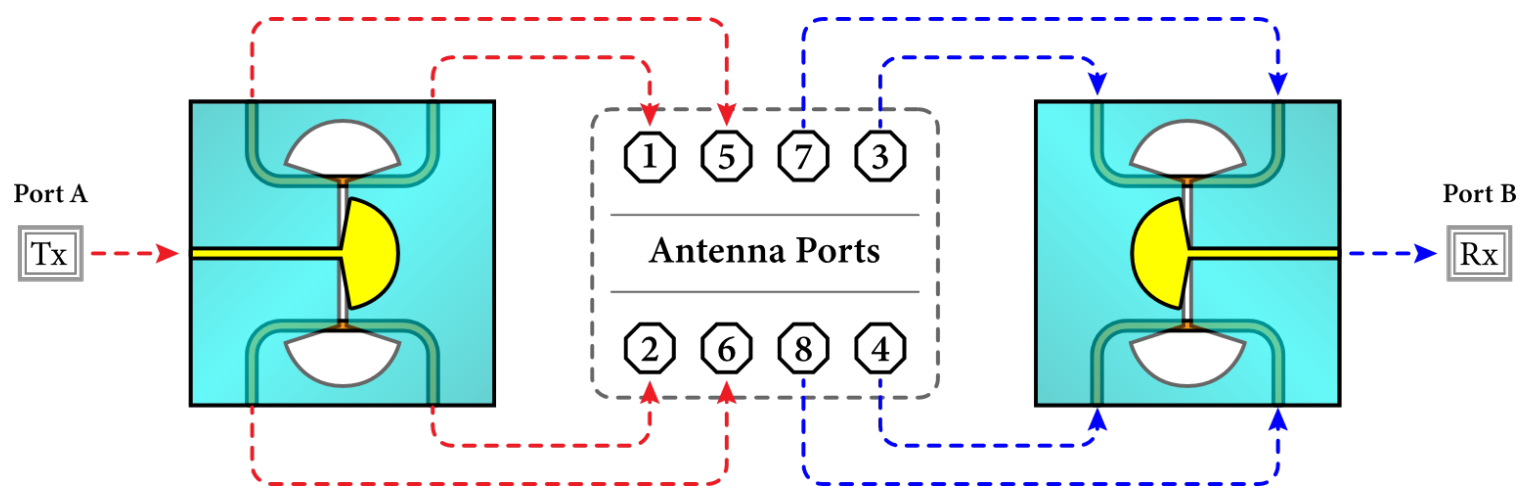

(c)

Figure 1: (a) the wideband dual-polarized Vivaldi array, (b) array top view with port numbering, and (c) array feeding network.

electric fields will combine destructively in near-field and constructively in far-field. This means that a null plane will be formed along the perpendicular bisector of the transmit antennas, in the near-field region, where the receive antennas are situated. Therefore, the transmit antennas cannot couple power directly to the receive antennas, which also means that, ideally, the receive antennas will be totally isolated from the transmit antennas. The opposite feeding of the antennas is provided by a 4-way out-of-phase power divider (or balun) that splits the input signal into four output signals of equal amplitudes, and two output signals will be phase inverted with respect to the other two. The proposed wideband balun will be discussed in the next section and the design of the wideband dual-polarized Vivaldi antenna will be discussed after.

\section{A. Wideband 4-way Balun}

First, referring to Figure 2, the proposed 4-way balun is a tri-layered structure with two RO4003C substrates having $\varepsilon_{r}=3.55$ and $h=203.2 \mu \mathrm{m}$. The balun is formed of a $50 \Omega$ input microstrip line terminated with a radial stub on the top layer, a slotline terminated with two radial stubs in the middle layer, and two $50 \Omega$ output microstrip lines on the bottom layer. The input and output microstrip lines can be placed on the same top layer, however, they were placed on separate layers to avoid any direct coupling between them which might degrade the balance of the output signals.

Now, if the power is injected in the input microstrip line through Port 1, it will couple to the slotline at its center where it will get divided equally between its left and right arms. Then at the end of each arm the divided power will couple to the corresponding output microstrip line. The electric fields at output Ports 4 and 5 will be inverted with respect to the input electric fields at Port 1, while the electric fields at output Ports 2 and 3 will be oriented in the same direction of the input electric fields. This also means that the signals at the output Ports 4 and 5 will be inverted with respect to those at Ports 2 and 3. This claim is confirmed by the plots in Figure 2(e) where a flat phase response are observed. Of course, in practice, we will observe more perturbations and imbalances in the phase and magnitude responses.

Also, by looking at the simulated S-parameters of the designed balun in Figure 2(d), it can be seen that the matching bandwidth at the input of the balun spans the frequency range from below $2 \mathrm{GHz}$ to beyond $20 \mathrm{GHz}$ (can reach $24.5 \mathrm{GHz}$ ). The wideband performance of the balun is attributed to the wideband behavior of microstrip-to-slotline transitions. Those 


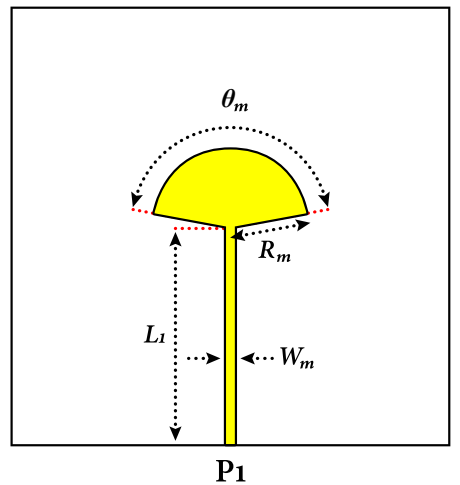

(a)

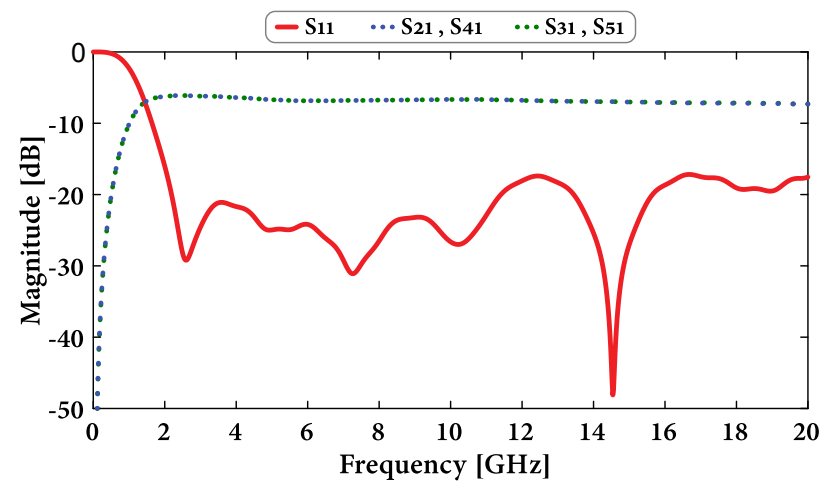

(d)
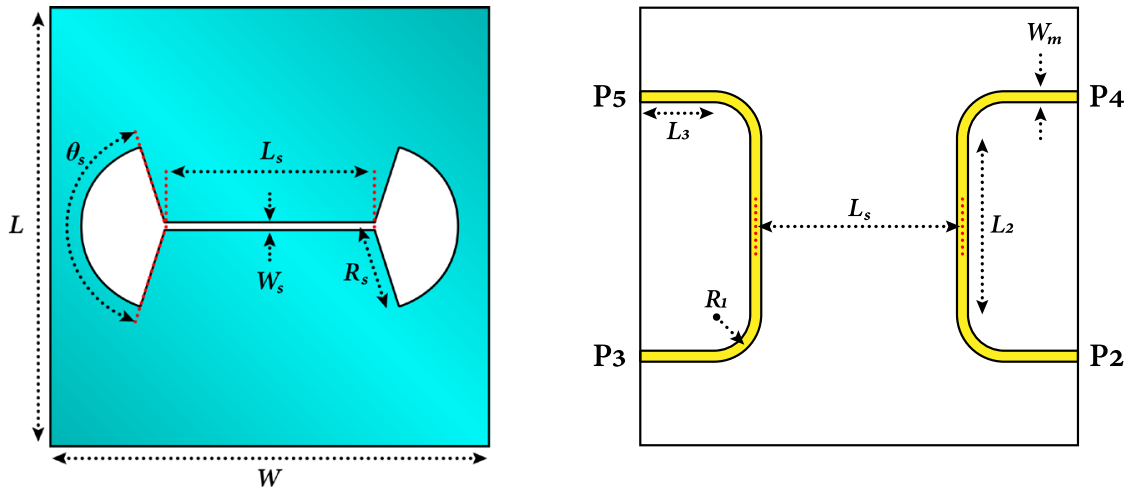

(b) (c)

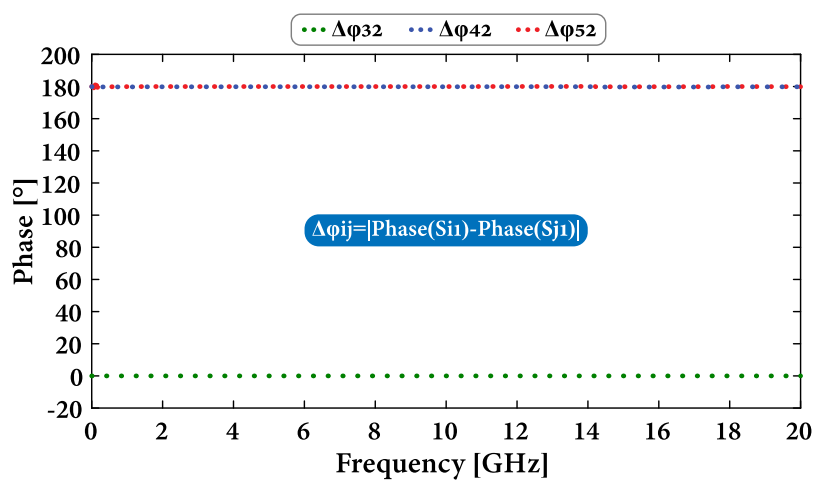

(e)

Figure 2: A 4-way balun (a) input microstrip line on top layer, (b) slotline in the middle layer, (c) output microstrip lines on the bottom layer, (d) simulated S-parameters, and (e) phase difference between output ports $\left\{W_{m}=0.45 \mathrm{~mm}, R_{m}=3.5 \mathrm{~mm}\right.$, $\theta_{s}=160^{\circ}, L_{1}=10 \mathrm{~mm}, L=20 \mathrm{~mm}, W=20 \mathrm{~mm}, W_{s}=0.25 \mathrm{~mm}, L_{s}=10 \mathrm{~mm}, R_{s}=3.75 \mathrm{~mm}, \theta_{s}=145^{\circ}, L_{2}=$ $8 \mathrm{~mm}, L_{3}=3.225 \mathrm{~mm}, R_{1}=2 \mathrm{~mm}$.

transitions can be matched over a decade bandwidth if they are terminated with radial stubs and tuned properly [11]. Moreover, the balun exhibits an insertion loss that increases from $6.24 \mathrm{~dB}$ at $2 \mathrm{GHz}$ to 7.32 at $20 \mathrm{GHz}$. However, note that $6 \mathrm{~dB}$ of the insertion loss are a result of the 4-way power division and are not actually lost in the balun. Finally, it was observed (but not shown here) that the balun lacks matching and isolation between its output ports. In fact, the balun can be viewed as a combination of three 3-port power dividers cascaded together, where each input and output microstrip line form a 3-port T-junction power divider with the slotline. And since 3-port devices cannot be lossless, reciprocal, and matched at all their ports at the same time, then the three Tjunctions, which are indeed lossless and reciprocal, cannot have matched and isolated output ports. Consequently, the balun will lack isolation and matching at the outputs.

\section{B. Wideband Dual-Polarized Vivaldi Antenna}

The Dual-polarized Vivaldi antennas can be formed either by crossing two single Vivaldi elements orthogonally at their centers [12], or by placing four single Vivaldi elements perpendicular to each other along their edges [13] in a boxlike configuration. Both approaches can achieve dual polarization with same system size, however, for printed circuit boards (PCBs), the first approach requires employing several mechanical operations, including cutting and soldering, to insert one antenna at the center of the other, and also to separate the feeding lines of both antennas. Moreover, since the feeding lines of both antennas are at proximity, the cross-polarization level can be degraded. On the other hand, the second approach uses a higher number of antennas and a more complex feeding network, but it can achieve a superior level of cross-polarization. Nonetheless, for both approaches, an external support is needed to hold and place the antennas precisely in their places, which might not be practical for many applications. In addition to that, due to their low thicknesses, PCB Vivaldi antennas are fragile and exhibit a lack of rigidity, and, in general, might not be suitable for applications operating in harsh environments. As an alternative solution, metal-machined Vivaldi antennas can be used instead. All-metal antennas are mechanically solid, selfsustained and do not require an external support, and they have a higher power-handling capability. In general, they will be many times thicker than PCB antennas, and they will be fed by coax-to-slot transition, which will replace the microstripto-slotline transition used in the PCB antennas.

In this work, an all-metal dual-polarized Vivaldi antenna is presented. The antenna is formed by crossing two Vivaldi elements at their centers as in Figure 1(a). And although this approach has an inferior cross-polarization level, however, in this context, the level of cross-polarization is not very critical because both polarizations will be used simultaneously to 


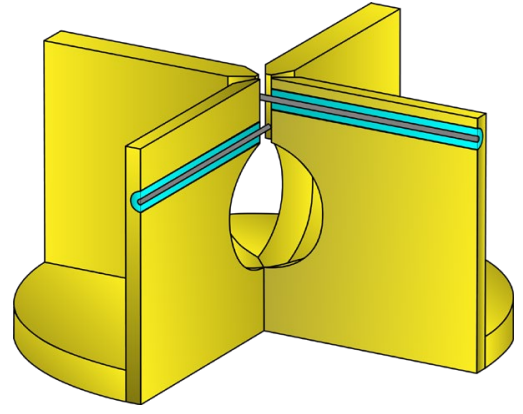

(a)

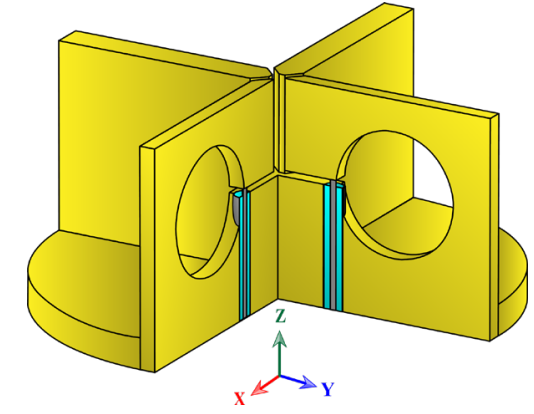

(b)

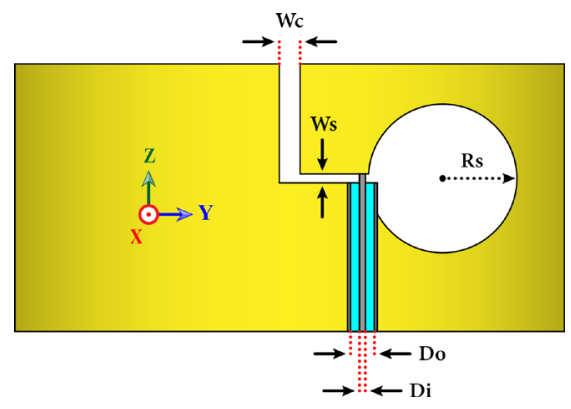

(c)

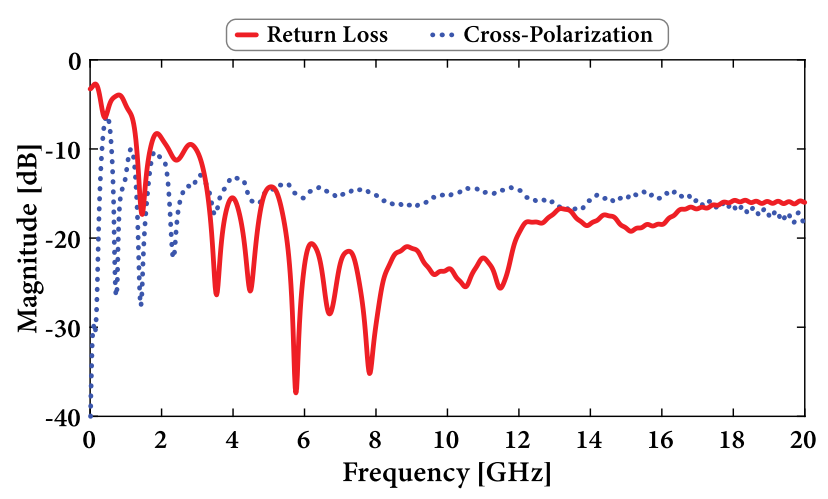

(d)

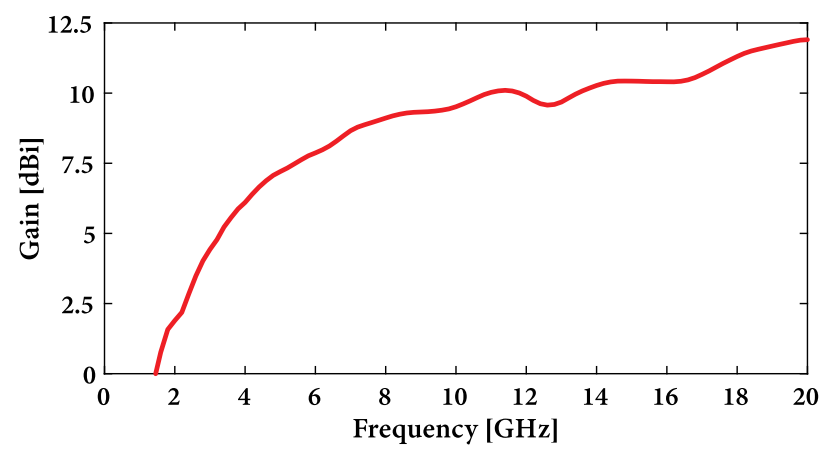

(e)

Figure 3: (a) conventional feeding of a dual-polarized Vivaldi antenna, (b) isometric view and (c) side view of the novel feeding, simulated (d) parameters and (e) gain of one polarization of the novel Vivaldi antenna $\left\{W_{c}=2.5 \mathrm{~mm}, W_{s}=1 \mathrm{~mm}\right.$, $\left.R_{s}=10 \mathrm{~mm}, D_{i}=0.94 \mathrm{~mm}, D_{o}=3.15 \mathrm{~mm}\right\}$.

transmit or receive the same signals. Also, using this approach can simplify the complicated feeding network of the array. Now, if two Vivaldi elements are crossed at their centers, their feeding coax-to-slot transitions will overlap. This requires alleviating the coax of one antenna above the other, as in Figure 3(a), which creates some problems:

- Firstly, due to the geometrical asymmetry between the orthogonal antennas, which is caused by alleviation of one coax, a difference in matching and radiation will be observed between the two orthogonal polarizations.

- Secondly, maximum antenna matching is achieved when the coax is placed closer to the matching stub. However, since one of the coaxs is placed further away from the stub, the corresponding polarization will suffer from a degraded matching as compared to the other one.

- Thirdly, the alleviated coax will stand in the way of the radiation generated by the orthogonal antenna, leading to its blockage or deformation especially at higher frequencies.

To overcome those drawbacks, we propose a new approach for feeding the dual-polarized Vivaldi antenna. The new feeding approach seeks to avoid the asymmetry between the orthogonal polarizations while achieving a wideband matching, and it targets to move the feeding points of the antenna from its center to its sides. This is achieved by bending the central slot to the sides of each antenna element as shown in Figures 3(b) and 3(c). The side slots can be matched to the corresponding coax lines independently from the central slot. This permits to manipulate the characteristics of the side slots to achieve wideband matching without degrading the radiation performance of the antenna. And by doing this, the geometrical symmetry between the orthogonal polarizations will be restored, and both polarizations will exhibit identical matching and radiation.

In simulation, the antennas were modeled as $5 \mathrm{~mm}$ thick aluminium sheets, and the inner and outer conductors of the coaxial lines were also modeled as aluminium cylinders having their radii equal to $0.47 \mathrm{~mm}$ and $1.575 \mathrm{~mm}$, respectively, while the dielectric stuffing of the coax was modeled as PTFE $\left(\varepsilon_{r}=2.1\right)$. In addition to that, the central and side slots have their widths equal to $2.5 \mathrm{~mm}$ and $1 \mathrm{~mm}$, respectively, and the matching stub has a radius of $10 \mathrm{~mm}$ And, moreover, the total size of the antenna $72.5 \times 72.5 \times$ $130 \mathrm{~mm}^{3}$. The simulated parameters of a single polarization of the proposed antenna are depicted in Figures 3(d) and 3(e). It can be seen that each polarization is matched from $3 \mathrm{GHz}$ to beyond $20 \mathrm{GHz}$, and that the level of cross-polarization is about $-15 \mathrm{~dB}$ throughout the matching bandwidth. The level of cross-polarization might seem not very good, but, once again, for our application the value is not very important because both polarizations will be used to transmit or receive the same signals at the same time. Finally, the gain of each polarization starts with $5 \mathrm{dBi}$ at $4 \mathrm{GHz}$, and then continues to increase with frequency until it reaches about $12 \mathrm{dBi}$ at 20 $\mathrm{GHz}$. 


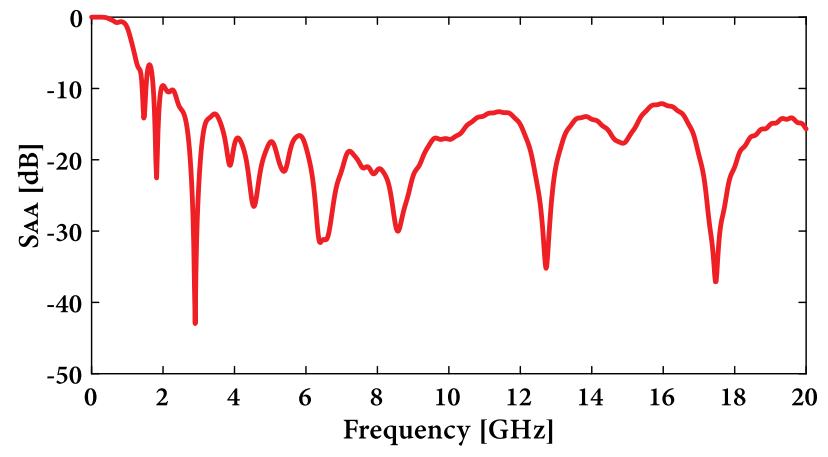

(a)

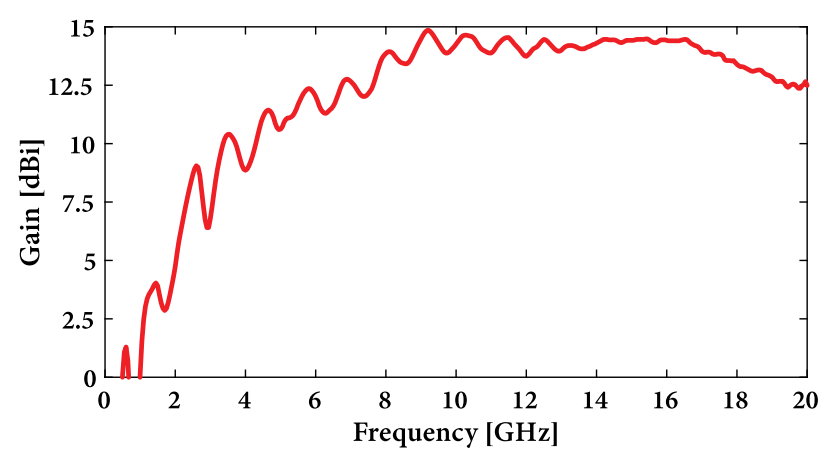

(c)

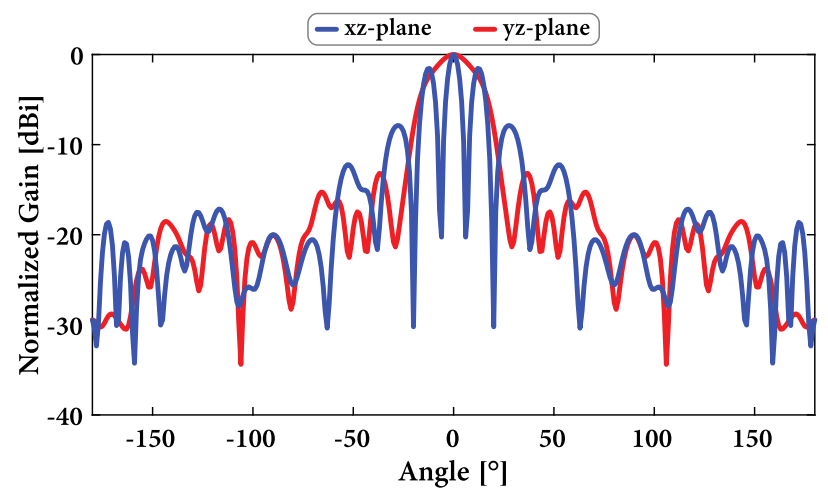

(e)

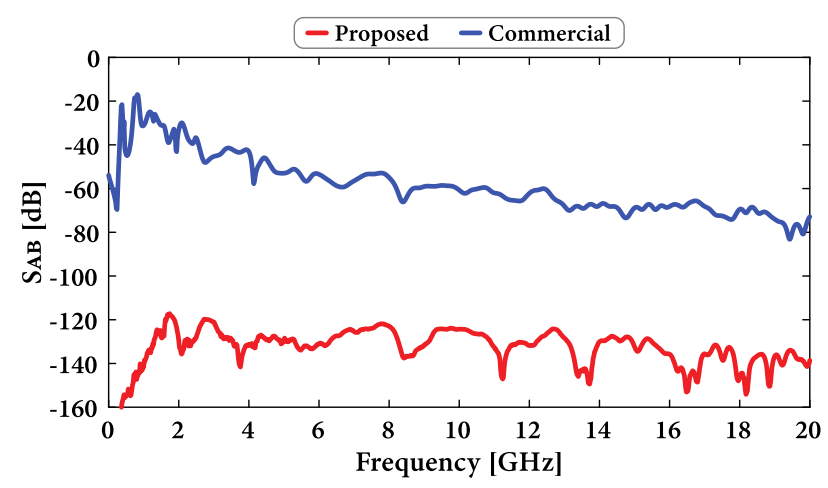

(b)

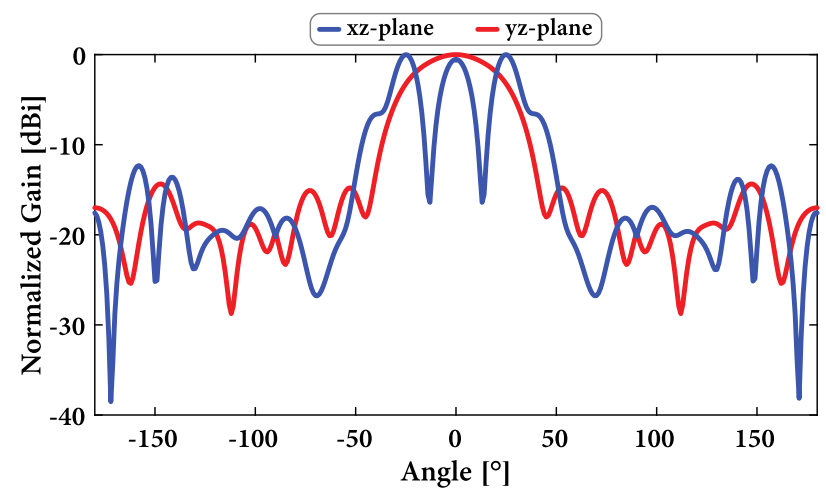

(d)

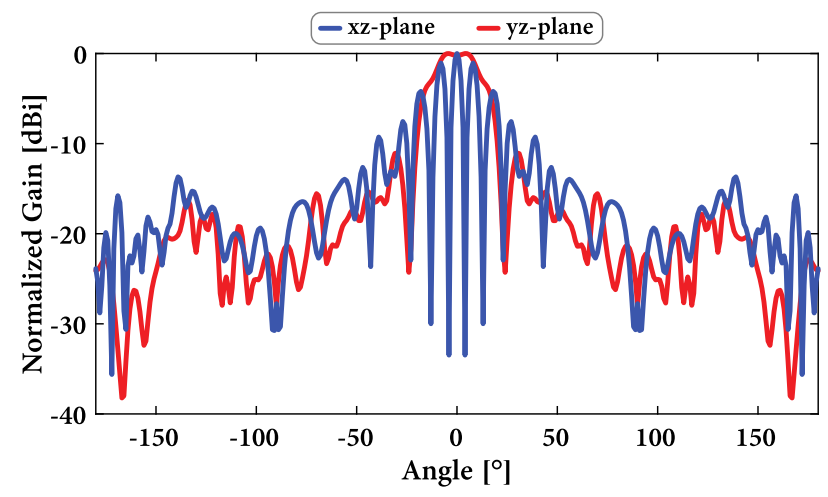

(f)

Figure 4: (a) array matching, (b) self-interference cancellation using the proposed balun and a commercial balun. (c) array gain, and radiation pattern plots at (d) $5 \mathrm{GHz}$, (e) $10 \mathrm{GHz}$, and (f) $15 \mathrm{GHz}$, respectively.

\section{ARRAY PERFormANCE}

Before The simulated S-parameters of the dual-polarized full-duplex array were obtained by numerically combining the simulated S-parameters of the four antennas together, with the simulated S-parameters of the 4-way balun. By looking at Figure 4(a) it can be seen that the array is matched from almost $2 \mathrm{GHz}$ to beyond $20 \mathrm{GHz}$. Also, Figure 4(b) shows that the array can achieve a very high level of self-interference cancellation $(\geq 120 \mathrm{~dB})$ using the proposed 4-way balun. This level of cancellation can be only achieved in simulation where it is assumed that the antennas are placed and aligned precisely, and where the simulated balun manages to achieve near-ideal characteristics (no amplitude and phase imbalances between the balun's output signals). However, in practice, there will always be some misplacement and misalignment between the antennas, and the amplitude and phase imbalances between the balun's output signals are expected to rise. Hence, the level of obtained cancellation is also expected to be lower.

In the light of that, a second type of feeding network, consisting of commercially available baluns and power dividers from Marki Microwaves, was also considered here for comparison. And in this case, the 4-way balun was formed by cascading, numerically, the S-parameters of a 2-way balun with two 2-way power dividers (Balun Model: BAL-0026 [14], Power Divider Model: PD-0R426 [15]). The indicated 
baluns and power dividers have their own phase and amplitude imbalances, and therefore they will form a 4-way balun with imbalances that are close to what we might get with a realized balun. The obtained level of cancellation using the commercial feeding network is also depicted in Figure 4(b) which increases from $40 \mathrm{~dB}$ at $2 \mathrm{GHz}$ to $80 \mathrm{~dB}$ at $20 \mathrm{GHz}$.

Now, concerning the far-field performance of the array, it can be noticed from Figure 4(c) that the gain of the array starts with $5 \mathrm{dBi}$ at $2 \mathrm{GHz}$ and then continues to increase with frequency until it reaches a peak value of $15 \mathrm{dBi}$ around 9 GHz. After that it maintains a value higher than $12.5 \mathrm{dBi}$ in the remaining part of the matching bandwidth. In addition to that, Figures 4(d), 4(e), and 4(f) depict some radiation pattern plots of the array at 5,10 , and $15 \mathrm{GHz}$, respectively. It is evident from those figures that there exists only one directive main lobe in the yz-plane and multiple directive lobes in the xz-plane. The additional directive lobes in the xz-plane, which are on the sides of the central lobe, are called grating lobes. And at least two grating lobes start to appear in the radiation pattern when the separation distance between the two radiating antennas is higher than a half-wavelength at the frequency of measurements. And the number of grating lobes starts to increase when the separation distance between the two radiating antennas becomes higher than a multiple of a half-wavelength at the measured frequency. The grating lobes are a drawback of the proposed array, and they can only be eliminated by reducing the separation distance between the antennas which is not feasible in this configuration, because the minimum separation distance is conditioned by the size of the individual antenna.

\section{CONCLUSION}

In this paper a wideband dual-polarized full-duplex antenna array is presented. The array is formed of four wideband dual-polarized Vivaldi antennas which are $90^{\circ}$ sequentially rotated with respect to one another A modified feeding was adopted for the Vivaldi antennas to ensure total symmetry between the orthogonal polarizations. Then the array was fed by a 4-way balun that can split the input signal into four output signals of equal amplitudes with two of them being phase inverted with respect to the other two. The simulated results indicate that the array is matched from 2 $\mathrm{GHz}$ to beyond $20 \mathrm{GHz}$ with an average gain of $11 \mathrm{dBi}$. Also, under ideal conditions, the array can achieve a high level of self-interference cancellation $(\geq 120 \mathrm{~dB})$, and this level is expected to be somewhere between 40 and $80 \mathrm{~dB}$ in practice. Finally, the radiation pattern plots reveal that the array can radiate highly directive beams with some grating lobes in one of its radiation planes. The grating lobes start to exist when the separation distance between the antennas is higher than a halfwavelength at the measured frequency. And although the distance is fixed, the grating lobes start to multiply in number as the frequency increases where the wavelength becomes smaller, and the separation distance becomes a multiple of a half-wavelength.

The array is currently being considered for fabrication and will be measured and characterized taking into account some enhancements: firstly, a way to reduce the size of the individual Vivaldi antenna is being investigated, which permits reducing the separation distance between the array elements and, therefore, reducing the level of grating lobes.
Moreover, adding a shield around the flares of the antennas is being considered as a way to enhance the radiation performance of the system (higher gain and lower side lobes). But this must not come at the expense of the bandwidth. Finally, it is also worth finding a way to shield the baluns. Shielding the baluns can help isolating them from one another and can prevent any coupling from the antennas to the baluns or vice versa, which in turn ensures that the level of selfinterference cancellation will not be deteriorated.

\section{REFERENCES}

[1] D. Bharadia, E. McMilin, and S. Katti, "Full Duplex Radios," in Proceedings of the ACM SIGCOMM 2013 conference on SIGCOMM, 2013, pp. 375-386.

[2] P. V. Prasannakumar, M. A. Elmansouri, and D. S. Filipovic, "Broadband Monostatic Simultaneous Transmit and Receive Reflector Antenna System," in 2017 IEEE International Symposium on Antennas and Propagation \& USNC/URSI National Radio Science Meeting. IEEE, 2017, pp. 553-554.

[3] E. A. Etellisi, M. A. Elmansouri, and D. S. Filipovic, "Wideband Monostatic Co-Polarized Co-Channel Simultaneous Transmit and Receive Broadside Circular Array Antenna," IEEE Transactions on Antennas and Propagation, vol. 67, no. 2, pp. 843-852, 2018.

[4] E. Everett, A. Sahai, and A. Sabharwal, "Passive Self-Interference Suppression for Full-Duplex Infrastructure Nodes," IEEE Transactions on Wireless Communications, vol. 13, no. 2, pp. 680-694, 2014.

[5] J. Wu, M. Li, and N. Behdad, "A Wideband, Unidirectional Circularly Polarized Antenna for Full-duplex Applications," IEEE Transactions on Antennas and Propagation, vol. 66, no. 3, pp. 1559-1563, 2018.

[6] W. F. Moulder, B. T. Perry, and J. S. Herd, "Wideband Antenna Array for Simultaneous Transmit and Receive (STAR) Applications," in 2014 IEEE Antennas and Propagation Society International Symposium (APSURSI). IEEE, pp. 243-244.

[7] P. V. Prasannakumar, M. A. Elmansouri, and D. S. Filipovic, "Wideband Decoupling Techniques for Dual-Polarized Bi-Static Simultaneous Transmit and Receive Antenna Subsystem," IEEE Transactions on Antennas and Propagation, vol. 65, no. 10, pp. 4991$5001,2017$.

[8] N.-A. Nguyen, V. H. Le, N. Nguyen-Trong, M. Radfar, A. Ebrahimi, K. Phan, and A. Desai, "Dual-polarized slot antenna for full-duplex systems with high isolation," IEEE Transactions on Antennas and Propagation, 2020.

[9] H. Nawaz and I. Tekin, "Dual-polarized, differential fed microstrip patch antennas with very high interport isolation for full-duplex communication," IEEE transactions on antennas and propagation, vol. 65, no. 12, pp. 7355-7360, 2017.

[10] J. Ha, M. A. Elmansouri, P. V. Prasannakumar, and D. S. Filipovic, "Monostatic Co-Polarized Full-Duplex Antenna with Left-or RightHand Circular Polarization," IEEE Transactions on Antennas and Propagation, vol. 65, no. 10, pp. 5103-5111, 2017.

[11] H. Hijazi, M. Le Roy, R. Lababidi, D. Le Jeune, and A. Pérennec, “4$40 \mathrm{GHz}$ In-Phase $/ 180^{\circ}$ Out-of-Phase Power Dividers with Enhanced Isolation," in 14th European Conference on Antennas and Propagation-EuCAP 2020, pp. 1-4.

[12] M. Sonkki, D. Sanchez-Escuderos, V. Hovinen, E. T. Salonen, and M. Ferrando-Bataller, "Wideband Dual-Polarized Cross-Shaped Vivaldi Antenna," IEEE Transactions on Antennas and Propagation, vol. 63, no. 6, pp. 2813-2819, 2015.

[13] M. Sonkki, S. Myllym“aki, J. Putaala, E. Heikkinen, T. Haapala, H. Posti, and H. Jantunen, "Dual Polarized Dual Fed Vivaldi Antenna for Cellular Base Station Operating at 1.7-2.7 GHz," International Journal of Antennas and Propagation, vol. 2017, 2017.

[14] Markimicrowave, "BAL-0026 Broadband Balun," https://www.markimicrowave.com/baluns/bal-0026.aspx

[15] Markimicrowave, "PD-0R426 2-Way Wilkinson Microwave/RF Power Divider / Combiner," https://www.markimicrowave.com/power-dividers/pd-0r426.aspx 